\title{
Disjoint homometric sets in graphs
}

\author{
Michael O. Albertson * \\ Smith College, Northampton, MA 01063, USA \\ Janos Pach ${ }^{\dagger}$ \\ City College, CUNY and Courant Institute, NYU, New York, NY 10012, USA \\ Michael E. Young $\ddagger$ \\ Iowa State University, Ames, IA 50010, USA
}

Received 28 June 2010, accepted 24 August 2010, published online 18 October 2010

\begin{abstract}
Two subsets of vertices in a graph are called homometric if the multisets of distances determined by them are the same. Let $h(n)$ denote the largest number $h$ such that any connected graph of $n$ vertices contains two disjoint homometric subsets of size $h$. It is shown that $\frac{c \log n}{\log \log n}<h(n)<\frac{n}{4}$, for $n>3$.
\end{abstract}

Keywords: Graph distances, homometric subsets, Golumb ruler.

Math. Subj. Class.: 05C12, 05C70

\section{Introduction}

It has been a well known fact in crystallography for over eighty years that the x-ray diffraction picture of a crystal depends on the set of vectors between its atoms [5]. As Pauling and Shapell found in 1930, the $x$-ray diffraction picture of the mineral bixbyite is consistent with two noncongruent atomic structures [8]. This motivated an interesting mathematical question: under what circumstances can a set be retrieved from its difference set. On the line, a partial answer was found by Piccard [9] in 1939. She claimed to prove that if two $n$-element sets of integers, $S$ and $T$, determine the same set of distinct differences, then $S$ and $T$ are congruent to each other. (A set that determines distinct differences is called

\footnotetext{
*Written posthumously.

† Work completed as Neilson Visiting Professor of Smith College, 2008.

$\ddagger$ Work completed while a Mellon Fellow at Smith College, corresponding author.

E-mail addresses: pach@cims.nyu.edu (Janos Pach), myoung@iastate.edu (Michael E. Young)
} 
a Golomb ruler [6].) It came as a big surprise that Bloom [4] found a counterexample to Piccard's theorem. The 6-element sets

$$
S=\{0,1,4,10,12,17\} \quad T=\{0,1,8,11,13,17\}
$$

determine the same set of differences. It is still not known whether there exist arbitrarily large sets with this property. If we allow repetition in the difference set, all pairs of sets with the same difference set were characterized by Rosenblatt and Seymour [10], using algebraic techniques.

Let $G=(V, E)$ be a simple connected graph on $n$ vertices. The distance between a pair of vertices $u, v \in V(G)$, denoted by $\operatorname{dist}(u, v)$ is the length of the shortest path from $u$ to $v$ in $G$. Let $S, T \subseteq V(G)$. The distance list of $S$ is the multiset of $\left(\begin{array}{c}|S| \\ 2\end{array}\right)$ pairwise distances of the vertices of $S$ in $G$. We say that $S$ and $T$ are homometric if their distance lists are equal.

In the special case when $G=C_{2 n}$, a cycle of length $2 n$, Lemke, Skiena, and Smith [7] proved that every $n$-element subset of the vertex set is homometric to its complement. In music theory, for $n=6$, this statement had been known for a long time as the Hexachordal Theorem. In the twelve-tone scale, any set of six notes determines the same multiset of differences. (For a historical account, consult [3], [2].) It is also known that the above theorem generalizes to vertex-transitive graphs [1].

Here we consider the following problem. What is the largest number $h$ such that any connected graph on $n$ vertices contains two disjoint homometric subsets, each of size $h$ ? We denote this largest number $h(n)$. In this paper we prove an upper and lower bound for $h(n)$.

\section{Bounds}

Theorem 2.1. $\frac{c \log n}{\log \log n} \leq h(n) \leq \frac{n}{4}$, for $n>3$.

Proof. We will prove the lower bound first. Let $G$ be a graph on $n$ vertices. Assume that there exists a vertex $v \in V(G)$, such that the degree of $v$ is at least $(\log n)^{3}$. Let $k=\log n$. All logarithms are base 2 in this proof. Partition the neighbors of $v$ into sets of size $k$. Each set has a distance list composed of 1 s and $2 \mathrm{~s}$. Therefore, there are at most $\left(\begin{array}{l}k \\ 2\end{array}\right)+1$ possibilities for each of the $k^{2}$ distance lists. Since $\left(\begin{array}{l}k \\ 2\end{array}\right)+1<k^{2}$, at least two of the distance lists must be the same. Hence, $k=2 \log n \leq h(n)$.

If it is the case that every vertex in $G$ has degree smaller than $d=(\log n)^{3}$, we will look to the diameter of $G$ to create our disjoint homometric sets. Letting $D$ denote the diameter of G, we have $n \leq d^{D}$. Thus, $D \geq \frac{\log n}{\log d}=\frac{\log n}{3 \log \log n}$. Since $D$ is the diameter, there exists an induced path in $G$ that has length $D$. Therefore, there exist two disjoint paths in $G$, both of which have length $\left\lceil\frac{D-1}{2}\right\rceil$; hence, $h(n) \geq\left\lceil\frac{D-1}{2}\right\rceil$.

For our upper bound we begin by defining a class of graphs called kites. We define an $(n, m)-k i t e$ as the graph constructed by taking a copy of $K_{n}$ and a path of length $m$ and adding an edge between a vertex in $K_{n}$ to a leaf vertex in the path.

Claim: Let $n=2(m-1)$, where $m$ is odd. An $(m, m-2)-k i t e$ has no homometric sets of size larger than $\frac{n}{4}$.

We prove this by contradiction. Assume there exists homometric sets $H_{1}, H_{2} \subset V(G)$ such that $\left|H_{1}\right|+\left|H_{2}\right|>\frac{n}{2}=m-1$. We know that $\left|H_{1}\right|+\left|H_{2}\right| \geq m+1$ since $\left|H_{1}\right|+\left|H_{2}\right|$ must be even. Therefore there exists a vertex in $H_{1} \cup H_{2}$ that is in the path and there exist 


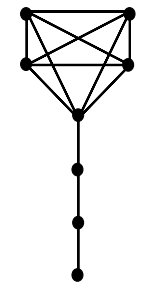

Figure 1: A $(5,3)-$ kite.

at least 3 vertices in $H_{1} \cup H_{2}$ that are in the complete graph. Let $v \in H_{1} \cup H_{2}$ be the vertex in the path that has the greatest distance to the complete graph. Without loss of generality we will assume that $v \in H_{1}$.

The only possible vertex that is in both $H_{1}$ and the complete graph is the vertex of the complete graph that is adjacent to the path. If there were another, say $u$, then it would not be adjacent to the path and in order to have a pair of vertices, $x, y \in H_{2}$ with $\operatorname{dist}(u, v)=$ $\operatorname{dist}(x, y)$, either $x$ or $y$ will have a greater distance than $v$ to the complete graph.

This implies that there are at least 2 vertices that are in both $H_{2}$ and the complete graph, where neither is adjacent to the path of $G$. Since $v$ is on the path, there must exist a vertex in $H_{2}$ that is also on the path. Therefore the largest distance in the distance list of $H_{2}$ will appear at least twice. The largest distance in $H_{1}$ is $\operatorname{dist}(v, w)$ where $w$ is the vertex in $H_{1}$ with the shortest distance to the complete graph. Since $v$ has the greatest distance to the complete graph, there can not be another pair of vertices whose distance is $\operatorname{dist}(v, w)$. Therefore, no such $H_{1}$ and $H_{2}$ exists. Hence, $h(n) \leq \frac{n}{4}$.

\section{References}

[1] T. A. Althuis and F. Göbel, Graph theoretic aspects of music theory, University of Twente, Dept. of Applied Math, Memoranda 1573, 2001.

[2] B. Ballinger, N. Benbernou, F. Gomez, J. O'Rourke and G. Toussaint, The Continuous Hexachordal Theorem, in: E. Chew, A. Childs and C-H. Chuan (eds.), Mathematics and Computation in Music, Second Internat'l Conf., MCM 2009, Springer-Verlag, Berlin, 2009, 11-20.

[3] S. K. Blau, The Hexachordal Theorem: A mathematical look at interval relations in twelve-tone composition, Math. Mag. 72 (1999), 310-313.

[4] G. S. Bloom, A Counterexample to a Theorem of S. Piccard, J. Comb. Theory Ser. A 22 (1977), 378-379.

[5] J. M. Cowley, Diffraction Physics, 2nd ed., North-Holland, Amsterdam, 1986.

[6] P. Gilbert and E. Postpischil, There Are No New Homometric Golomb Ruler Pairs with 12 Marks or Less, Journal of Experimental Mathematics 3 (1994), 147-152. 
[7] P. Lemke, S. S. Skiena and W. D. Smith, Reconstructing sets from interpoint distances, Discrete and Computational Geometry: The Goodman-Pollack Festschrift, vol. 25 of Algorithms Combin., Springer, Berlin, 2003, 597-631.

[8] L. Pauling and M. D. Shappell, Zeits. Krist. 75 (1930), 128-142.

[9] S. Piccard, Sur les ensembles des distances des ensembles des points d'un espace euclidien, Mém. Univ. Neuchátel 13 (1939), 78.

[10] J. Rosenblatt and P. D. Seymour, The structure of homometric sets, SIAM Journal of Algorithms and Discrete Mathematics 3 (1982), 343-350. 